\section{Comparison of internal jugular vein dilation between Valsalva maneuver and proximal internal jugular vein compression}

\author{
Hyeonjoo Seong ${ }^{1}$, Bora Kang ${ }^{2}$, Giwoon Kim ${ }^{3}$ \\ 'Department of Emergency Medicine, Ajou University School of Medicine, Suwon, Korea \\ ${ }^{2}$ Department of Emergency Medicine, Seoul National University Bundang Hospital, Seongnam, Korea \\ ${ }^{3}$ Department of Emergency Medicine, Soon Chun Hyang University Bucheon Hospital, Bucheon, Korea
}

Objective The Valsalva maneuver is recognized as an effective method to dilate the internal jugular vein (IJV). However, this maneuver cannot be performed in many cases, such as children and unconscious patients. The aim of this study was to evaluate the effectiveness of proximal IJV compression, which can easily be performed, regardless of patient cooperation.

Methods Healthy adult volunteers were recruited from tertiary hospital employees. Basic anatomic and physiologic data were collected. The subjects lay down as if they were undergoing IJV catheter insertion, in the supine position with their necks turned 30 degrees to the left. The main outcome was the cross-sectional area (CSA) of the distal IJV as measured by ultrasound in four stages. The first stage was sham without any maneuver. The second was Valsalva maneuver, the third was digital IJV compression, and the fourth was digital compression accompanied by simultaneous Valsalva maneuver.

Results A total of 41 volunteers were enrolled. Twenty-six (63.41\%) were male with an average age of $28.15 \pm 2.85$ years. Mean height was $170.74 \pm 8.66 \mathrm{~cm}$ and mean neck circumference was $35.28 \pm 3.87 \mathrm{~cm}$. The mean CSA-IJV was $1.06 \pm 0.36 \mathrm{~cm}^{2}$ without any maneuver. It increased to $1.34 \pm 0.45 \mathrm{~cm}^{2}$ with Valsalva maneuver $(\mathrm{P}<0.001)$, to $1.26 \pm 0.41 \mathrm{~cm}^{2}$ with digital compression $(P<0.001)$, and to $1.41 \pm 0.47 \mathrm{~cm}^{2}$ with the two maneuvers combined $(P=0.01)$.

Conclusion Digital proximal IJV compression effectively dilates the distal IJV. When performed simultaneously with the Valsalva maneuver, the effect was enhanced.

Keywords Catheterization; Jugular veins; Valsalva maneuver; Ultrasound
elSSN: 2383-4625

Received: 10 June 2016

Revised: 20 September 2016

Accepted: 26 September 2016

Correspondence to: Giwoon Kim Department of Emergency Medicine, Soon Chun Hyang University Bucheon Hospital, 170 Jomaru-ro, Wonmi-gu, Bucheon 14584, Korea

E-mail: flyingguy0202@daum.net

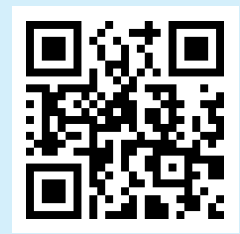

How to cite this article:

Seong H, Kang B, Kim G. Comparison of internal jugular vein dilation between Valsalva maneuver and proximal internal jugular vein compression. Clin Exp Emerg Med 2016;3(4):193-196

This is an Open Access article distributed under the terms of the Creative Commons Attribution Non-Commercial License (http:// creativecommons.org/licenses/by-nc/4.0/). 


\section{INTRODUCTION}

Internal jugular vein catheterization (IJC) is useful in emergency departments (ED) for monitoring hemodynamic status and for infusion of fluid or nutrients. As compared to subclavian vein catheterization, IJC has fewer complications such as pneumothorax or hemothorax, and thus is more often recommended.'

As first described in the 1980s, the use of bedside ultrasonography by emergency physicians in the ED has many advantages. For example, it can be performed 24 hours a day and is easily accessible. In addition, it can contribute to improved patient satisfaction and quality of emergency care, as well as a reduction in the length of ED stays. ${ }^{2}$

In recent years, USG-guided IJC has been widely used. However, it may be difficult to perform in cases of small cross-sectional area (CSA) of the internal jugular vein (IJV) with USG-guidance (e.g., in hypovolemic patients). To overcome this limitation, methods such as the Valsalva maneuver, Trendelenburg position, and passive leg elevation have been applied to dilate the IJV. ${ }^{3-5}$ The Valsalva maneuver is performed through moderately forceful attempted exhalation against a closed airway, usually done by closing one's mouth while simultaneously expiring against a closed glottis. This elicits a cardiovascular response, increasing venous pressure and resulting in venous dilation.

Bellazzini et al. ${ }^{6}$ reported that the Valsalva maneuver could dilate the IJV by $38 \%$, or even more if combined with the Trendelenburg position. However, the Valsalva maneuver may be difficult to perform in unconscious patients or uncooperative patients such as children.

To our knowledge, this study provides the first description regarding digital proximal compression of the IJV. We aimed to investigate the effectiveness of digital proximal compression of the IJV on CSA-IJV alone and in combination with Valsalva maneuver.

\section{METHOD}

\section{Study design}

We conducted an experimental study to assess the effects of compressing the IJV on CSA-IJV. The study was approved by the hospital's institutional review board, and informed consent was waived because the procedure is not invasive, not painful, and not time-consuming (AJIRB-MED-DE0-15-239).

\section{Study population}

We enrolled healthy volunteers for study participation. Employees of a tertiary hospital ED were recruited. We then excluded participants with a history of IJC or neck surgery.

\section{Study protocol}

Each patient lay down in the supine position, and the diameter of his or her neck was measured. The measurement point of the CSA-IJV was at the top point of the triangle created by the clavicle and the sternal and clavicular heads of the sternocleidomastoid muscle. By ultrasonography (L15W probe; Zonare Medical Systems, Mountain View, CA, USA), this was approximately at the level of the thyroid cartilage prominence.

Three examiners independently measured the CSA-IJV. These examiners were all board-certified emergency physicians with academic experience with emergency ultrasonography. The average measurement on maximal diameter, our main outcome, was calculated by the ultrasonography machine (Fig. 1).

For each participant and examiner, the CSA-IJV was measured in four different stages. For the first stage, the CSA-IJV was measured with the participant at rest without any maneuver. For the second stage, the participant was instructed to perform the Valsalva maneuver by holding his or her breath and compressing the lower abdomen for 5 seconds, after which the CSA-IJV was measured. For the third stage, our main focus of the study, the participant was instructed to breathe normally while their proximal IJW was compressed by the examiner's index finger (Fig. 2). The compression point was identified as the lower border of the triangle that is delineated by the sternocleidomastoid and clavicle. The CSA-IJV was measured within 20 seconds of compression. The last stage combined techniques used in the second and third stages, such that digital proximal compression and the Valsalva maneuver were performed simultaneously. The CSA-IJV was mea-

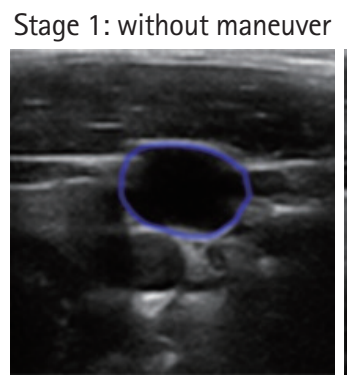

Stage 2: with Valsalva

Stage 3: with compression Stage 4: with Valsalva and compression
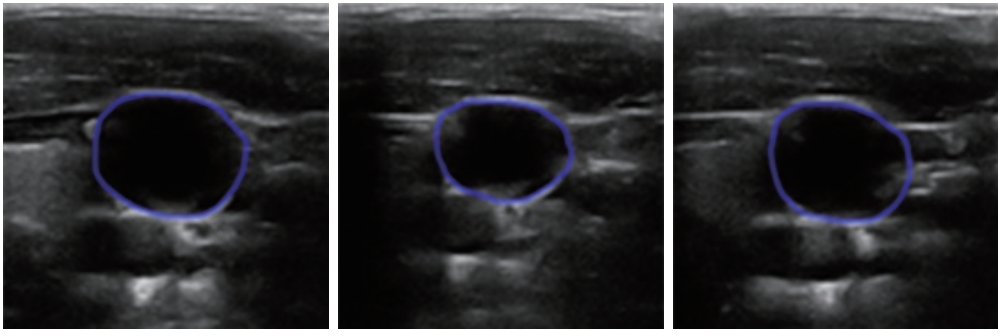

Fig. 1. Ultrasound view of distal internal jugular vein at each stage. Cross-sectional area was calculated by measuring the two axes of the ellipse. 
sured within 20 seconds of compression. A five-minute resting period was allowed between each stage.

\section{Statistical analysis}

Variables were expressed as means and standard deviations. To test the normality, the Kolmogorov-Smirnov test was performed. Paired ttest was performed for comparison between groups. P-values $<0.05$ were considered significant. All analyses were performed using IBM SPSS ver. 22.0 (IBM Corp., Armonk, NY, USA) and R ver. 3.1.2 (R Foundation for Statistical Computing, Vienna, Austria; http://www.R-project.org/) software packages. The sample size was calculated from preliminary data using the 'sample size' library with $\mathrm{R}$ package. To detect a $0.2 \mathrm{~cm}^{2}$ differences with a power of 0.8 and a significance level of 0.05 from data with a standard deviation of 0.4 using a paired ttest, the required number of cases was calculated to be 39 .

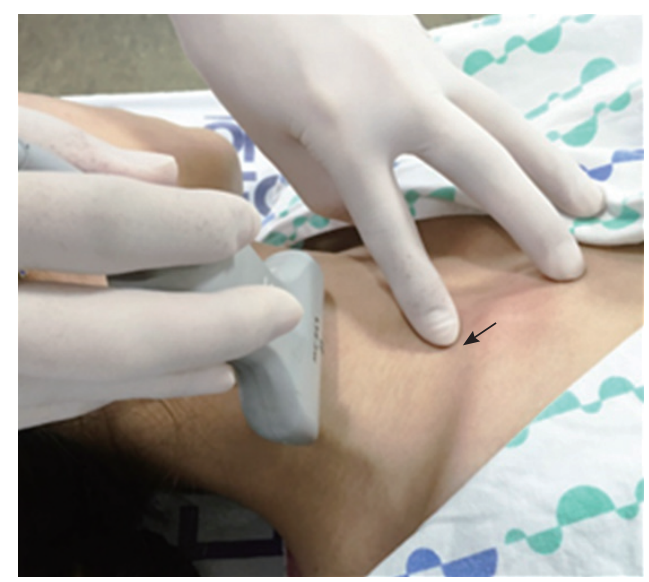

Fig. 2. Example of performing the 3rd stage of measurement. The left index finger of the examiner is pointed at the proximal internal jugular vein (arrow), where the sternocleidomastoid muscle and clavicle meet. At the same time, the examiner's right hand holds the ultrasound probe, which is gently positioned over and perpendicular to the internal jugular vein.

Table 1. Characteristics of selected participants

\begin{tabular}{lc}
\hline Variable & Value \\
\hline Age & $28.15 \pm 2.85$ \\
Sex & \\
$\quad$ Male & $26(63.41)$ \\
Female & $15(36.59)$ \\
Weight $(\mathrm{kg})$ & $67.61 \pm 13.39$ \\
Height $(\mathrm{cm})$ & $170.74 \pm 8.66$ \\
Neck circumference $(\mathrm{cm})$ & $35.28 \pm 3.87$ \\
Neck vertical length $(\mathrm{cm})$ & $12.73 \pm 2.11$ \\
Tonsillectomy history & $2(4.88)$ \\
\hline
\end{tabular}

Values are presented as mean \pm standard deviation or number $(\%)$.

\section{RESULTS}

\section{Baseline characteristics}

A total of 41 volunteers were enrolled. Twenty-six (63.41\%) were male, and the mean age was $28.15 \pm 2.85$ years. Mean height was $170.74 \pm 8.66 \mathrm{~cm}$ and mean neck circumference was $35.28 \pm 3.87$ $\mathrm{cm}$. One volunteer had a history of neck surgery and was excluded from the study. Two participants had tonsillectomies prior to the study, but were included nonetheless. The general characteristics of participants are presented in Table 1.

\section{Comparison of outcomes}

The means and standard deviations of CSA-IJV following each maneuver are listed in Table 2.

The CSA-IJV with the Valsalva maneuver was significantly greater than that of the Stage 1 control $(1.34 \pm 0.45$ vs. $1.06 \pm 0.36$ $\mathrm{cm}^{2}$, respectively, $\left.\mathrm{P}<0.001\right)$. The $C S A-I J V$ with digital proximal compression of the IJV was significantly different from that of the control (1.26 \pm 0.41 vs. $1.06 \pm 0.36 \mathrm{~cm}^{2}$, respectively, $\left.\mathrm{P}<0.001\right)$. Moreover, the CSA-IJV under Valsalva maneuver was significantly larger than the CSA-IJV with proximal compression of the IJV $\left(1.34 \pm 0.45\right.$ vs. $1.26 \pm 0.41 \mathrm{~cm}^{2}$, respectively, $\left.P=0.0112\right)$.

The CSA-IJV during simultaneous digital proximal compression and the Valsalva maneuver was significantly larger than that of digital compression alone $\left(1.41 \pm 0.47\right.$ vs. $1.26 \pm 0.41 \mathrm{~cm}^{2}$, respectively, $\mathrm{P}<0.001)$. It was also larger than that of the Valsalva maneuver alone, but without achieving statistical significance (1.41 \pm 0.47 vs. $1.34 \pm 0.45 \mathrm{~cm}^{2}$, respectively, $P=0.062$ ).

\section{DISCUSSION}

To our knowledge, this is the first study to show the effectiveness of digital proximal compression of the IJV on the CSA-IJV.

For many years, the blind IJC method has been used in the ED. However, blind IJC might lead to serious complications, such as arterial puncture, nerve injury, or failure. As a result, ultrasoundguided IJC is currently the method of choice.

Table 2. Descriptive statistics for cross-sectional area by each method

\begin{tabular}{ccccc}
\hline & Stage 1 & Stage 2 & Stage 3 & Stage 4 \\
\hline Mean \pm standard $\left(\mathrm{cm}^{2}\right)$ & $1.06 \pm 0.36$ & $1.34 \pm 0.45$ & $1.26 \pm 0.41$ & $1.41 \pm 0.47$ \\
\% changes compared to stage 1 & - & 26.4 & 19.0 & 33.0 \\
Median $(I 0 R, 25 \%-75 \%)\left(\mathrm{cm}^{2}\right)$ & 1.02 & 1.3 & 1.23 & 1.35 \\
& $(0.85-1.26)$ & $(1.05-1.57)$ & $(1.00-1.46)$ & $(1.05-1.60)$ \\
\% changes compared to stage 1 & - & 27.4 & 20.5 & 32.5 \\
\hline
\end{tabular}

Stage 1, without any maneuver; stage 2, Valsalva maneuver; stage 3, digital compression; stage 4, digital compression with Valsalva maneuver. $I Q R$, interquartile range. 
Many modifications have been suggested to improve the success of IJC. Wang et al. ${ }^{7}$ reported that the angle of the neck or ultrasonography-guided method could affect the success rate of IJC. Armstrong et al. ${ }^{8}$ showed that use of the Trendelenburg position up to 30 degrees enlarges the CSA-IJV and increases the success rate of IJC. Other methods, such as compression of the liver or placement of a pillow under the head, have been investigated. On the other hand, compression of the carotid artery or a pillow under the shoulder have been found to lower the success rate of IJC. ${ }^{9,10}$

The Valsalva maneuver could be an ideal method to increase the CSA-IJV, and thereby facilitate IJC, but it requires the cooperation of patients. Therefore, it definitely could have limitations in pediatric or unconscious patients. The Trendelenburg method can be performed on uncooperative patients, but requires a tilting table, which may not be available in all EDs around the world.

Given these limitations, proximal compression of the IJV, which could be easily done in any ED, might be useful in performing IJC. In this study, even though the CSA from proximal compression of the IJV was slightly smaller than that following the Valsalva maneuver, it was much larger than the control CSA-IJV. The amount of dilation achieved could effectively aid proper insertion of an IJV line. Moreover, the combination of digital proximal compression and the Valsalva maneuver resulted in the largest CSA-IJV. This suggests that ED physicians could perform proximal compression alone in uncooperative patients to improve the odds of successful IJC. In cooperative patients, they could combine this technique with the Valsalva maneuver to help maximize the CSA-IJV.

We have a few limitations to discuss. First, in this study, we simply measured the CSA-IJV with each maneuver. Due to ethical considerations, we could not perform the IJC and compare the success rates of this invasive procedure. However, we can safely infer that a larger CSA-IJV would enhance the odds of success in IJC. Second, we could not standardize the digital pressure on the proximal IJV. In reality, it would be hard or nearly impossible for clinicians to exert the same pressure. Finally, our statistical significance could be strengthened by enlarging the study sample size.

In summary, digital proximal compression of the IJV dilated the IJV, and this could have a positive effect on the success of IJC. Further clinical trials are needed to validate this technique.

\section{REFERENCES}

1. Jeong $\mathrm{HH}$, Yoon $\mathrm{JH}, \mathrm{Oh} \mathrm{S}$, et al. A quantitative analysis of the relation between the clavicular tilt angle and subclavian central venous catheter misplacement. Clin Exp Emerg Med 2014; 1:114-9.

2. Ahn C, Kim C, Kang BS, Choi HJ, Cho JH. Variation of availability and frequency of emergency physician-performed ultrasonography between adult and pediatric patients in the academic emergency department in Korea. Clin Exp Emerg Med 2015;2:16-23.

3. Verghese ST, Nath A, Zenger D, Patel RI, Kaplan RF, Patel KM. The effects of the simulated Valsalva maneuver, liver compression, and/or Trendelenburg position on the cross-sectional area of the internal jugular vein in infants and young children. Anesth Analg 2002;94:250-4.

4. Clenaghan S, McLaughlin RE, Martyn C, McGovern S, Bowra J. Relationship between Trendelenburg tilt and internal jugular vein diameter. Emerg Med J 2005;22:867-8.

5. Kim JT, Kim HS, Lim YJ, et al. The influence of passive leg elevation on the cross-sectional area of the internal jugular vein and the subclavian vein in awake adults. Anaesth Intensive Care 2008;36:65-8.

6. Bellazzini MA, Rankin PM, Gangnon RE, Bjoernsen LP. Ultrasound validation of maneuvers to increase internal jugular vein cross-sectional area and decrease compressibility. Am J Emerg Med 2009;27:454-9.

7. Wang R, Snoey ER, Clements RC, Hern HG, Price D. Effect of head rotation on vascular anatomy of the neck: an ultrasound study. J Emerg Med 2006;31:283-6.

8. Armstrong PJ, Sutherland R, Scott DH. The effect of position and different manoeuvres on internal jugular vein diameter size. Acta Anaesthesiol Scand 1994;38:229-31.

9. Manikappa S, Cokis C. Assessment of internal diameter and cross-sectional area of right internal jugular vein pre-induction and post-intubation. Anaesth Intensive Care 2005;33:381-3.

10. Parry G. Trendelenburg position, head elevation and a midline position optimize right internal jugular vein diameter. Can J Anaesth 2004;51:379-81.

\section{CONFLICT OF INTEREST}

No potential conflict of interest relevant to this article was reported. 